PROF. ADRIANA MÓNICA TORRES (Orcid ID : 0000-0002-8133-0137)

Article type : Original Article - Kidney

\title{
TIME EVOLUTION OF METHOTREXATE-INDUCED KIDNEY INJURY. A COMPARATIVE STUDY \\ BETWEEN DIFFERENT BIOMARKERS OF RENAL DAMAGE IN RATS
}

\author{
María Julia SEVERIN, Romina Valeria CAMPAGNO, Anabel BRANDONI, \\ Adriana Mónica TORRES*
}

Pharmacology Area, Faculty of Biochemical and Pharmaceutical Sciences. National University of Rosario. CONICET. Argentina

Short title: A new biomarker for methotrexate renal failure

All the authors have no conflict of interest to declare

*To whom correspondence should be addressed

Name: Adriana Mónica Torres, Ph.D. Professor of Pharmacology

Address: Suipacha 531

City: Rosario Postcode: 2000

Country: Argentina

Tel: 0054/341/4393400

Email: adtorres@fbioyf.unr.edu.ar 


\section{ABSTRACT}

Methotrexate (MTX) is commonly used in the treatment of malignant diseases and autoimmune and chronic inflammatory disorders. Along with its effective therapeutic power, MTX has adverse effects on the kidneys. Discovery of new biomarkers is required to improve the early detection of renal damage and optimize the effectiveness of treatments. The aim of this study was to evaluate the time course of MTX-induced nephrotoxicity and to compare the urinary excretion of the organic anion transporter 5 (uOat5) with alterations in other markers of renal function, and to elucidate the possible molecular mechanisms involved in uOat5. Animals were exposed to a unique dose of MTX ( $80 \mathrm{mg} / \mathrm{kg}$ body weight, intraperitoneal). Experiments were carried out at day 2, 4, 8 or 14 after MTX administration. Markers of renal damage, such as creatinine and urea plasma levels, urinary activity of alkaline phosphatase, microalbuminuria, urinary excretion of Neutrophil Gelatinase-Associated Lipocalin (UNGAL) and histopathology were evaluated. Renal Oat5 and its presence in different urine fractions abundance were assessed by Western blotting. uOat 5 was significantly increased 2 days after MTX treatment, before than any alteration in other parameters of kidney injury or renal morphology occurred. UNGAL showed an inverted pattern of urinary excretion compared to uOat5. Exosomal pathway is involved in the urinary excretion of Oat5 and depends on the degree of damage induced by MTX. These experimental data allow proposing uOat5 as a potential noninvasive biomarker for early detection of MTX-induced nephrotoxicity.

Key words: methotrexate; kidney injury; biomarkers; organic anion transporter 5

\section{INTRODUCTION}

Methotrexate (MTX) is one of the most widely used antimetabolite chemotherapeutic to treat malignancies, rheumatologic conditions and other chronic inflammatory disorders. MTX is worldwide employed due to several characteristics, such as its low price, high response rates and therapeutic continuity, and its availability and versatility in dosage and routes of administration ${ }^{1}$. Unfortunately, MTX may produce renal damage both at high and low doses. Several long-term

This article is protected by copyright. All rights reserved. 
observational studies indicate that the most common reason of discontinuing MTX regimens is its toxicological profile rather than lack of efficacy ${ }^{2-5}$. MTX causes renal dysfunction mainly due to direct pharmacological toxicity against renal tubules and/or precipitation of MTX or its metabolites ${ }^{3-}$ ${ }^{5}$. MTX undergoes renal excretion primarily by glomerular filtration and tubular secretion ${ }^{3,4}$. Since MTX is secreted by the renal tubules, its elimination in urine may be reduced by the concomitant administration of other renal secreted drugs. Thus, increased MTX toxicity may occur more often in patients with reduced creatinine clearance and those receiving combined therapy ${ }^{6}$.

The organic anion transporter $5(0 a t 5, S / c 22 a 19)$ is exclusively located in the apical membranes of proximal tubule cells. Oat5 has been defined as a probenecid-sensitive organic anion/dicarboxylate exchanger, which can transport ochratoxin A, dehydroepiandrosterone sulfate and estrone-3-sulfate, and can be inhibited by bumetanide, furosemide, penicillin $\mathrm{G}$ and by some sulfate conjugates ${ }^{7}$. Our group was pioneering in detecting Oat5 in urine and proposing its urinary excretion (uOat5) as an early indicator of proximal tubule damage in ischemic acute kidney injury, in nephropathy induced by mercury or cisplatin, and in the renal damage associated with vascular calcification ${ }^{8-13}$. Recently, we have also found a dose-related increase in Oat5 urinary abundance in MTX-induced renal damage ${ }^{14}$. The traditional blood and urinary markers used for diagnosis and prognosis of kidney injury are insensitive and nonspecific, which not only delays diagnosis and intervention, but also underestimates the degree of injury ${ }^{15,16}$. Incorporation of new biomarkers for kidney damage into clinical practice might optimize the effectiveness of MTX treatments and the prognostic assessment of a given treatment.

The aim of this study was to evaluate the time course of MTX-induced renal damage and to compare uOat5, with alterations in traditional markers of renal injury (such as creatininemia, uremia, urinary alkaline phosphatase (AP), microalbuminuria (MAU) and renal histology), and with the urinary excretion of Neutrophil Gelatinase-Associated Lipocalin (UNGAL, a novel biomarker of renal damage ${ }^{17}$. To analyze the possible molecular mechanisms involved in the urinary elimination

This article is protected by copyright. All rights reserved. 
of Oat5 after different times of MTX administration, its expression in renal tissue and in different urine fractions have been assessed.

\section{RESULTS}

Animals were exposed to $80 \mathrm{mg} / \mathrm{kg}$ b.w. i.p. of MTX and experiments were performed after 2, 4, 8 and 14 days of the injection of the agent or vehicle.

As it is shown in Figure 1, uOat5 was significantly increased 2 days after MTX injection. Interestingly, this biomarker appearance in urine continued elevated until the fourth day of treatment. From day 8, Oat5 levels in urine returned to basal values.

Different traditional parameters of renal function were measured in plasma and urine in comparison with time evolution of uOat5. Figure 2 shows urea (A) and creatinine (B) plasma levels, as well as creatinine clearance values (C). A significant alteration in M4D group was observed for all these parameters, which reflects renal dysfunction on the fourth day after the injection of MTX. Renal function ameliorated by the eighth and fourteenth day.

The histological evaluation of kidneys from Control rats and treated with MTX at each time point is shown in Figure 3. In M2D group (Figure 3B) no significant histological damages were observed compared to control kidneys. In contrast, on the fourth day after MTX injection, considerable damage of renal cells was observed, mainly with a flattened tubular epithelium, tubular dilatation and loss of the brush border membrane in proximal tubules (Figure 3C). M8D group showed an improvement of the nephron's architecture with the presence of luminal debris (Figure 3D). On day 14, renal morphology was fully recovered compared to controls (Figure 3E). The tubular injury scores are shown in Figure 3F.

Urinary AP, MAU and UNGAL were also assessed to evaluate renal injury in comparison with uOat5 (Figure 4). No alteration of urinary activity of AP (Figure 4A) was observed. On the other hand, MAU (Figure 4B) was increased at the fourth day post-treatment. In contrast, uNGAL (Figure 4C)

This article is protected by copyright. All rights reserved. 
decreased on the second day after MTX treatment, remained low at the fourth and eight day, and then started to return to basal levels.

To understand the origin of Oat5 found in urine, both Oat5 expression and localization were evaluated in renal tissue (total homogenates and apical membranes) and in urine fractions (EXO and SN) at the time points corresponding to alterations in uOat5 $\left(2^{\text {nd }}\right.$ and $4^{\text {th }}$ day after MTX treatment). Figure $5 \mathrm{~A}$ shows that no alterations were found in the expression of Oat5, in neither renal homogenates nor in apical membranes. Immunohistochemistry studies (Figure 5B) showed a strong Oat5 labeling associated with the apical plasma membrane in proximal tubule cells. Oat5 immunostaining was consistent with the results observed by Western blotting in each experimental group. The analysis of Oat5 abundance in urine fractions showed a significant increase in EXO (similar percentage to that observed in urine), without modifications in SN after 2 days of MTX treatment. On the contrary, Oat5 expression was increased in $\mathrm{SN}$ and decreased in EXO, respectively after 4 days of the drug administration (See Figure 6A). To better understand the mechanism of exosomal excretion of Oat5 in the presence of different degrees of MTX-induced renal damage, another experimental group of animals was exposed to $360 \mathrm{mg} / \mathrm{kg}$ b.w., i.p. of MTX and experiments were performed after 2 days, since it has been previously demonstrated a dramatic renal injury with this MTX dose ${ }^{14}$. In this group of animals, a marked increase of Oat5 abundance was observed in total urine and in the soluble urine fraction, while Oat5 expression decreased in EXO (Figure 6B).

\section{DISCUSSION}

MTX, an antifolate drug, is an effective antineoplastic agent when administered in a high dose. When given in low doses, it is an effective drug for rheumatoid arthritis ${ }^{1,2,6}$. Side effects of MTX treatment are well known and described ${ }^{3}$. The most significant risk factor for MTX-toxicity is

impaired renal function ${ }^{18,19}$. At this point, it is important the early diagnosis of kidney injury in order to take corrective measures during MTX treatment. Nowadays, serum creatinine remains the gold-

This article is protected by copyright. All rights reserved. 
standard to renal failure diagnosis. Nevertheless, this is a suboptimal marker of early renal dysfunction and their levels are often not reflective of the glomerular filtration rate, since serum concentration is greatly influenced by numerous non-renal factors ${ }^{17,20}$. Ongoing investigations focus on new biomarkers that can identify renal cells injury when functional impairment is still minimal $16,17,20$. In this regard, renal tubule transporters have gained interest in the biomarker field; for instance, aquaporin 2 has been detected in rat and human urine, and recently proposed as a potential biomarker for gentamicin-induced nephrotoxicity ${ }^{21}$ and the urinary excretion of the $\mathrm{Na}^{+} / \mathrm{H}^{+}$ exchanger isoform 3 has also been suggested as an acute renal failure marker ${ }^{22}$. In this connection and attending the fact that the mortality and morbidity associated with acute kidney injury remains high despite several therapeutic advances, our laboratory group was pioneering in detecting Oat5 in urine and in postulating uOat5 as a noninvasive early biomarker of proximal tubule injury in preclinical models of renal and extra-renal pathologies ${ }^{8-14}$.

In the present study, the goal was to evaluate the time course of uOat5 to strengthen the hypothesis of its possible role as a more sensitive biomarker of MTX-induced renal damage compared to traditional, routinely used biomarkers of nephrotoxicity. To accomplish that objective, a time-response study was performed and uOat5 was measured and contrasted to the conventional plasma biomarkers and histological changes after a single dose of $80 \mathrm{mg} / \mathrm{kg}$ b.w. i.p. of MTX. Studies were performed after 2, 4, 8 and 14 days of MTX treatment. On the second day, uOat5 was significantly increased as compared with control values while no modifications in any traditional renal function markers and no relevant histological findings had still been observed. In contrast, plasma levels of urea and creatinine, and creatinine clearance were significantly altered at the fourth day post MTX treatment, accompanied by an important impairment of the tubular architecture observed by histological studies. By this time point, uOat5 remained elevated, but in a lower percentage as compared with the second day. MAU, which is considered an early marker of kidney injury ${ }^{23}$, increased significantly only on the fourth day post treatment, in the same manner as 
plasma urea, which reflects that it is not an early marker for MTX-induced nephrotoxicity. Urinary AP levels displayed no alterations, showing a lack of sensitivity for the renal damage produced by MTX.

In this work, it was also assessed UNGAL, which has been investigated extensively as a promising early biomarker of kidney injury ${ }^{17,24}$. Under normal conditions, filtered NGAL is almost completely reabsorbed by the proximal tubules resulting in minimal UNGAL levels, but it is highly expressed in damaged renal tubules and can be quickly detected in urine ${ }^{25}$. There are several clinical studies and reviews remarking the potential role of the increase urinary excretion of NGAL as a reliable diagnostic and prognostic biomarker of acute renal injury of different etiologies ${ }^{26}$. Surprisingly, the present results showed that not only UNGAL does not increase but decreases at all the time points evaluated after MTX administration. MTX has anti-inflammatory and immunosuppressive properties by leading a decrease in proinflammatory cytokines production ${ }^{27}$. Moreover, it is described that MTX suppresses NF-KB activation by releasing adenosine, contributing even more to MTX immunosuppressive effects ${ }^{28}$. In relation to this, it has also been reported that NGAL may be upregulated in a NF-KB-dependent manner ${ }^{29}$. In this regard, it is possible to consider that the presence of MTX may downregulate NGAL synthesis or liberation as consequence of the suppression of NF-KB activation.

Further studies will be performed to compare the behavior of uOat5 with other novel biomarkers of acute kidney injury in this experimental model. To our knowledge, there are few reports about the implementation of new biomarkers in urine to diagnose MTX-induced acute kidney injury. After different MTX treatments in rats, some studies employed novel biomarkers to assess renal damage, besides measuring the traditional ones (such as blood urea nitrogen and plasma creatinine). They reported the increase of KIM-1 in renal tissue ${ }^{30,31}$ and an augmented urinary NAG ${ }^{32}$ and Cys $\mathrm{C}^{33}$. On the other hand, Carvalho Pedrosa et al. ${ }^{34}$ showed a direct correlation between MTX serum levels and urinary KIM-1 levels 24 hours after MTX infusion in pediatric patients. In the same way, plasma Cys $\mathrm{C}$ was useful to evaluate the renal performance before and after the infusion of HDMTX in pediatric patients with acute lymphoblastic leukemia ${ }^{35}$. Recently, a

This article is protected by copyright. All rights reserved. 
study proposed to incorporate serum levels of Cys $\mathrm{C}$ as a predictive marker of the elimination of MTX in high-dose regimens in adult patients with central nervous system primary lymphoma ${ }^{36}$.

In summary, Oat5 urinary excretion significantly increased after 2 days of MTX treatment when the other markers of renal function (uremia, creatininemia, creatinine clearance, urinary alkaline phosphatase activity, microalbuminuria, and renal morphology) were not modified. Thus, it is worth noting that uOat5 has the sensitivity to anticipate renal damage, enhancing its interesting behavior as an early biomarker. Coca and Parikh ${ }^{37}$ have defined five phases of biomarker development for detection of acute kidney injury. Our study is in phase 1 , also called discovery phase. The data obtained in the present work together with those previously reported by Severin et al. ${ }^{14}$ identifies promising directions of phase 1 that lay the foundations to continue with phase 2 of these studies aimed to validate uOat5 as an early biomarker of MTX renal damage.

In order to understand the mechanism by which Oat5 reaches urine, renal expression of Oat5 and its abundance in different urine fractions were evaluated in animals treated with MTX. No alterations were observed in renal homogenates or in apical membranes in all the experimental groups, despite Oat5 increases in urine. These results were consistent with immunohistochemistry studies. Since renal expression of this protein was not modified at any treated group, it is possible to suggest that after this toxicant insult, uOat5 depends on a selective apical pathway. Urinary exosomes are nanovesicles $(50-100 \mathrm{~nm})$ derived from kidney cells and urinary tract and have been postulated as a potential source of specific biomarkers of renal diseases ${ }^{38,39}$. Previous studies of our laboratory suggested the implication of the exosomal pathway as a mechanism by which Oat5 would appear in urine ${ }^{13}$. In order to determine if the exosomal pathway would participate as the main mechanism of Oat5 urinary excretion after MTX treatment, the protein abundance of Oat5 in urinary EXO and in the soluble fraction was evaluated. The data obtained suggest that uOat5 is mediated by the exosomal pathway when the renal structure and function are still preserved, as observed after two days of MTX treatment (M2D group). In addition, it was corroborated that the uOat5 behaves as an early biomarker of MTX renal injury and that these vesicles would be a good source of it. On the

This article is protected by copyright. All rights reserved. 
contrary, when the renal damage was established (four days of treatment, M4D group) and all renal parameters were altered, Oat5 was excreted in the soluble fraction. This data would lead to the hypothesis that MTX toxicity might affect intracellular signaling mechanisms that are involved in the exosomal pathway and therefore in the incorporation of Oat5 into these vesicles. To corroborate this hypothesis, another experimental group of animals were exposed to a highly nephrotoxic dose of MTX (M360 2D group) ${ }^{14}$. In this new group, a marked increase of Oat5 abundance was observed in total urine and in the soluble fraction, while Oat5 expression decreased in EXO. According to the data presented, it is possible to conclude that the mechanism of uOat5 would depend on the degree of injury produced by MTX. The mechanisms by which exosomal cargo is sorting are still unknown. Some authors suggested that the NF-KB pathway could be involved in exosomal protein expression, leading to an alteration in exosomal pathway ${ }^{40}$. Therefore, suppression of NF-KB by adenosine release due to MTX treatment might be, at least in part, related to the exosome synthesis and liberation. More work is needed to elucidate this thought.

In conclusion, it is plausible to propose uOat5 as a potential noninvasive early biomarker of MTX-induced renal injury that it could be measured in total urine and in urinary exosomes with good analytic sensitivity. This work opens new perspectives in using the uOat5 as part of a panel of biomarkers of nephrotoxicity.

\section{MATERIAL AND METHODS}

\subsection{Chemicals and reagents}

Chemicals were purchased from Sigma (St. Louis, MO, USA) and were analytical grade pure. The rabbit polyclonal antibody against Oat5 was kindly provided by Prof. N. Anzai (Department of Pharmacology, Graduate School of Medicine, Chiba University, Japan). The molecular ruler (Kaleidoscope Prestained Standards of molecular mass) was purchased from Bio Rad Laboratories (Hercules, CA, USA).

This article is protected by copyright. All rights reserved. 


\subsection{Animals and treatments}

Male Wistar rats aged 110-130 days from Central Bioterium of Faculty of Biochemical and Pharmaceutical Sciences, National University of Rosario were used throughout the study. Animals were cared for in accordance with the principles and guidelines for the care and use of laboratory animals, recommended by the National Academy of Sciences and published by the National Institute of Health (NIH publication $8^{\text {th }}, 2011$ ). All experimental procedures were approved by the Faculty of Biochemical and Pharmaceutical Sciences Institutional Animal Care and Use Committee (Res. $\left.N^{\circ} 484 / 2015\right)$. During the experiments, animals were housed in an environment of constant temperature $\left(21-23^{\circ} \mathrm{C}\right)$ and humidity with regular light cycles of 12 hours and with free access to tap water and food. This study was carried out using only male rats in order to avoid any sex hormones influences on the data to be obtained. This work was carried out after the set-up of the working conditions (dose and time points chosen for the experiments). Criteria for inclusion: age, sex and weight of the animals. Exclusion criteria: animals showing signs of discomfort, distress or stress related to incorrect manipulation, not related to renal damage.

MTX was administrated by intraperitoneal (i.p.) injection of MTX, as was previously reported in our laboratory and by other authors ${ }^{14,41-44}$. Intraperitoneal injection is used for small species for which intravenous access is challenging, such as Wistar rats, and it can be used to administer large volumes of fluid safely ${ }^{45}$.

The animals were randomly divided and treated with a single injection of MTX ( $80 \mathrm{mg} / \mathrm{kg}$ of body weight, b.w., i.p.) on day 0. Experiments were carried out in different groups: at day 2 (M2D, $n$ $=4)$, day $4(M 4 D, n=4)$, day $8(M 8 D, n=4)$ or day $14(M 14 D, n=4)$ after MTX administration. Corresponding controls to each experimental group injected with vehicle ( $1 \mathrm{~mL} / \mathrm{saline} \mathrm{kg}$, b.w., i.p.) were processed. As all the tested parameters in control groups $(2,4,8$ and 14 days after vehicle administration) were similar, it was decided to consider them as one unified group (Control, $n=10$ ) to facilitate the analysis of the results, as previously described ${ }^{12,46}$. This set of animals was used for biochemical determinations and preparation of urine samples, total homogenates and apical 
membranes from kidneys, and another set of rats ( $n=4$ for each experimental groups) was used for histopathological and immunohistochemical studies. An additional group of animals treated with a single dose of $360 \mathrm{mg} / \mathrm{kg}$ b.w., i.p. of MTX 2 days before the experiments was used (M360 2D, n=4).

The rats were placed in metabolic cages $24 \mathrm{~h}$ before experiments without food to improve urine sample quality, as previously described ${ }^{14,47}$ and with access to water ad libitum through urine collection periods. On the day of the experiment, urine was collected. The urinary volume was determined gravimetrically and urinary flow rate $\left(\mathrm{U}_{\mathrm{f}}\right)$ was expressed as $\mathrm{mL} / 24 \mathrm{~h} / 100 \mathrm{~g}$ b.w. Then, the animals were anesthetized with sodium thiopental (70 $\mathrm{mg} / \mathrm{kg}$ b.w., i.p.); blood samples were obtained by cardiac puncture and kidneys were removed. After surgical procedures, the animals received euthanasia using anesthetic overdose and death was confirmed by bilateral thoracotomy.

\subsection{Biochemical determinations}

Urine samples were centrifuged at $1000 \times \mathrm{g}$ for $10 \mathrm{~min}$ to discard whole cells and cellular detritus, and the supernatants were used for evaluating AP activity, MAU, creatinine $\left(\mathrm{Cr}_{U}\right)$, as well as the protein abundance of Oat5 and NGAL. Plasma samples were used to measure urea (urea p), and creatinine levels (creatinine p). Urea $\mathrm{p}$ and creatinine $\mathrm{p}$, as well as $\mathrm{Cr}_{U}$, AP activity and MAU were determined spectrophotometrically, employing commercial kits (Wiener Laboratory, Rosario, Argentina). Urinary protein levels of Oat 5 and NGAL were evaluated by Western blotting. AP, MAU, Oat5 and NGAL in urine were related to urinary creatinine concentration to correct for variations in urine production as previously described ${ }^{10-14}$. Creatinine clearance $\left(\mathrm{Cl}_{\mathrm{cr}}\right)$ was calculated by the conventional formula: $\mathrm{Cl}_{\mathrm{Cr}}=\left(\mathrm{Cr}_{\mathrm{U}} \times \mathrm{U}_{\mathrm{f}}\right) /$ creatinine $\mathrm{p}$.

\subsection{Preparation of total homogenates and apical membranes from kidneys}

Total homogenates and apical membranes were prepared from 4 animals from each experimental group. Apical membranes from kidneys were obtained by Mg/EGTA precipitation. Kidneys were removed and the renal tissue was minced and homogenized in $30 \mathrm{~g} / 100 \mathrm{~mL}$ of ice-cold $50 \mathrm{mM}$

This article is protected by copyright. All rights reserved. 
mannitol, $5 \mathrm{mM}$ EGTA, $10 \mathrm{mM}$ HEPES-Tris $\mathrm{HCl}$ buffer ( $\mathrm{pH}$ 7.40) and $1 \mathrm{mM}$ phenylmethylsulfonyl fluoride (PMSF) for $5 \mathrm{~min}$ at top speed in an homogenizer (Glas-Col ${ }^{\circledR}$, Indiana, USA). From this preparation, total renal homogenates were obtained, and aliquots were taken and stored at $-80{ }^{\circ} \mathrm{C}$ until use. Then, $\mathrm{MgCl}_{2}$ was added to the rest of the renal homogenate to a final concentration of 12 $\mathrm{mM}$ and a set of differential centrifugation steps was performed as previously described. Finally, the apical membranes were resuspended in $50 \mathrm{mM}$ mannitol, $10 \mathrm{mM}$ Hepes-Tris ( $\mathrm{pH} 7.50)$ and $1 \mathrm{mM}$ PMSF. Aliquots of the membranes were stored immediately at $-80^{\circ} \mathrm{C}$ until use. Protein quantification of samples was performed using the method of Lowry with some modifications, as previously described ${ }^{10-14,41}$.

\subsection{Isolation of urinary exosomes}

Urinary exosomes were isolated from urine as previously described by Bulacio et al. ${ }^{13}$. In brief, $24 \mathrm{~h}$ urine samples from different experimental groups were centrifuged at $17,000 \times \mathrm{g}$ for $15 \mathrm{~min}$ at $4{ }^{\circ} \mathrm{C}$ to remove large membrane fragments, whole cells and other debris. The final pellets (exosome fraction, EXO) obtained after the second ultracentrifugation at 200,000 $\times \mathrm{g}$ for $1 \mathrm{~h}$ at $4{ }^{\circ} \mathrm{C}$ (OptimaTM XL-235 $100 \mathrm{~K}$ Ultracentrifuge, Beckman Coulter, Inc.; Ti 80 rotor) were resuspended in an appropriate volume of isolation solution and frozen at $-80{ }^{\circ} \mathrm{C}$ until used. An aliquot of the supernatant ( $\mathrm{SN}$, also typed as soluble fraction) obtained after the second ultracentrifugation at $200,000 \times \mathrm{g}$ for $1 \mathrm{~h}$ at $4^{\circ} \mathrm{C}$ was also stored at $-80^{\circ} \mathrm{C}$. Both samples (EXO and SN) were used to assess Oat5 abundances. Protein contents were measured, and samples were prepared for electrophoresis and immunoblotting as previously described ${ }^{10-14}$.

\subsection{Electrophoresis and immunoblotting}

Oat5 protein abundances were assessed by electrophoresis and Western blotting in total renal homogenates ( $20 \mu \mathrm{g}$ of protein), apical membranes (16 $\mu \mathrm{g}$ of protein), total urine $(10 \mu \mathrm{L}), \mathrm{EXO}(16 \mu \mathrm{g}$ of protein) and SN (16 $\mu \mathrm{g}$ of protein) samples as previously described ${ }^{10-14}$. NGAL protein levels were

This article is protected by copyright. All rights reserved. 
also determined in total urine samples $(10 \mu \mathrm{L})$. Ponceau Red was used to verify equal protein loading and transfer between lanes as previously described ${ }^{12,20}$. In this connection, Romero-Calvo et al. ${ }^{48}$ clearly demonstrated that routine quantification of Ponceau staining is validated as an alternative to actin blotting. Nitrocellulose membranes were incubated with a non-commercial rabbit polyclonal antibody against rat Oat5 or with a commercial mouse monoclonal antibody against rat NGAL (Thermo Fisher Scientific, Rockford, IL, USA). The specificity of Oat5 antibody has been described elsewhere ${ }^{7}$. Blots were processed for detection using a commercial kit (Pierce ${ }^{\mathrm{TM}}$ ECL Western Blotting Substrate, Thermo Fisher Scientific, Rockford, IL, USA). A densitometric quantification of the Western blot signal intensity of membranes was performed (Gel-Pro Analyzer ${ }^{\mathrm{TM}}$ Media Cybernetics, Silver Spring, MD, USA). For densitometry of immunoblots, samples from treated rats were run on each gel with corresponding control samples and normalized to Ponceau Red. The mean of the control value was set as $100 \%$. Treated groups protein abundances were expressed as percentage of the mean control value for that gel. Each value represents mean \pm standard error from experiments carried out in triplicate on four different tissue derived samples or urine derived samples for each experimental group. The protein abundances of Oat5 and NGAL in the urine samples were expressed as optical density (O.D., arbitrary units) related to urinary creatinine concentration [(O.D./10 uL)/Cru] in order to correct for variations in urine production as previously reported ${ }^{10,11,14,46,49}$.

\subsection{Histopathological and immunohistochemistry studies}

For histopathological and immunohistochemistry studies, kidneys were perfused with saline, followed by perfusion with periodate-lysine-paraformaldehyde solution $\left(0.01 \mathrm{M} \mathrm{NalO}_{4}, 0.075 \mathrm{M}\right.$ lysine, $0.0375 \mathrm{M}$ phosphate buffer, with $2 \%$ paraformaldehyde, $\mathrm{pH} 6.20$ ) through a cannula introduced in the abdominal aorta, as previously described ${ }^{10-14}$. Some sections were processed for routine staining with hematoxylin-eosin to evaluate renal injury. The severity of tubular injury was considered as percent of tubules of the section showing a given tubular alteration (tubular dilatation/flattening, loss of brush border, cellular detachment, intraluminal nuclei and debris), and

This article is protected by copyright. All rights reserved. 
was graded as follows: 0 , less than $5 \%$; $1,5-33 \%$; $2,34-66 \%$ and 3, over $66 \%$, as previously described ${ }^{50}$. Another set of paraffin section was used for Oat5 immunohistochemistry. To this purpose, a non-commercial polyclonal antibody against Oat5 (diluted 1:100) was used. Pictures were obtained with an optical microscope and are representatives of samples obtained from four animals from each experimental group.

\subsection{Statistical analysis}

Statistical differences between groups were evaluated using the unpaired Student's t-test or multiple comparisons with one-way ANOVA followed by the Newman-Keuls test. $p$ values of less than 0.05 were considered statistically significant. The values are expressed as the means \pm standard error (SE). For these analyses, GraphPad 6 software (San Diego, California, USA) was used. Student's t-test: $\left({ }^{*}\right) p<0.05$ versus Control. Newman-Keuls test: (a) $p<0.05$ versus Control, (b) $p<0.05$ versus M2D, (c) $p<0.05$ versus M4D, (d) $p<0.05$ versus M8D, (e) $p<0.05$ versus M14D.

\section{ACNKOWLEDGEMENTS}

This work was supported by the following grants: Agencia Nacional para la Investigación Científica y Tecnológica (ANPCyT) PICT 2017: Nº936, Consejo Nacional de Investigaciones Científicas y Técnicas (CONICET) PIP 2015-2017: N00460, and Universidad Nacional de Rosario (UNR) PIP 2016-2019, BIO 479. The authors thank to Prof. N. Anzai (Department of Pharmacology, Graduate School of Medicine, Chiba University, Japan) for kindly providing Oat5-specific antibodies and Mrs Alejandra Martínez (Area Morfología, Facultad de Ciencias Bioquímicas y Farmacéuticas, U.N.R.) for her collaboration in the present work. The authors also thank Wiener Lab Argentina for analytical kits. The authors declare that they have no conflict of interest.

This article is protected by copyright. All rights reserved. 


\section{REFERENCES}

1.- Tornero Molina J, Ballina García FJ, Calvo Alén J, Caracuel Ruiz MA, Carbonell Abelló J, López Meseguer A, et al. Recommendations for the use of methotrexate in rheumatoid arthritis: Up and down scaling of the dose and administration routes. Reumatol. Clin. 2014; 11:3-8.

2.- Borchers AT, Keen CL, Cheema GS, Gershwin ME. The use of methotrexate in rheumatoid arthritis. Semin. Arthritis Rheum. 2004; 34:465-83.

3.- Widemann BC, Adamson, PC. Understanding and managing methotrexate nephrotoxicity Oncologist 2006; 11:694-703.

4.- Green MR, Chamberlain MC. Renal dysfunction during and after high-dose methotrexate. Cancer Chemother. Pharmacol. 2009; 63:599-604.

5.- Perazella MA, Moeckel GW. Nephrotoxicity from chemotherapeutic agents: clinical manifestations, pathobiology, and prevention/therapy. Semin. Nephrol. 2010; 30:570-81.

6.- Patanè M, Ciriaco M, Chimirri S, Ursini F, Naty S, Grembiale RD, et al. Interactions among low dose of methotrexate and drugs used in the treatment of rheumatoid arthritis. Adv. Pharmacol. Scie. 2013; 313858, 8 pages.

7.- Anzai N, Jutabha $\mathrm{P}$, Enomoto A, Yokoyama H, Nonoguchi H, Hirata $T$ et al. Functional characterization of rat organic anion transporter 5 (S/c22a19) at the apical membrane of renal proximal tubules. J. Pharmacol. Exp. Ther. 2005; 315:534-44.

8.- Di Giusto G, Anzai N, Endou H, Torres AM. Oat5 and NaDC1 protein abundance in kidney and urine after renal ischemic reperfusion injury. J. Histochem. Cytochem. 2009; 57:17-27.

9.- Di Giusto G, Torres AM. Organic anion transporter 5 renal expression and urinary excretion in rats exposed to mercuric chloride: a potential biomarker of mercury-induced nephropathy. Arch. Toxicol. 2010; 84:741-49.

10.- Hazelhoff MH, Bulacio RP, Torres AM. Organic anion transporter 5 renal expression and urinary excretion in rats with vascular calcification. Biomed. Res. Int. 2013; 283429, 10 pages.

This article is protected by copyright. All rights reserved. 
11.- Bulacio RP, Torres AM. Organic anion transporter 5 (Oat5) renal expression and urinary excretion in rats treated with cisplatin: a potential biomarker of cisplatin-induced nephrotoxicity. Arch. Toxicol. 2013; 87:1953-62.

12.- Bulacio RP, Torres AM. Time course of organic anion transporter 5 (Oat5) urinary excretion in rats treated with cisplatin: a novel urinary biomarker for early detection of drug induced nephrotoxicity. Arch. Toxicol. 2015; 89:1359-69.

13.- Bulacio RP, Anzai N, Ouchi M, Torres AM. Organic anion transporter 5 (Oat5) urinary excretion is a specific biomarker of kidney injury: evaluation of urinary excretion of exosomal Oat5 after n-acetylcysteine prevention of cisplatin induced nephrotoxicity. Chem. Res. Toxicol. 2015; 28:1595-602.

14.- Severin MJ, Trebucobich MS, Buszniez P, Brandoni A, Torres AM. The urinary excretion of an organic anion transporter as an early biomarker of methotrexate-induced kidney injury. Toxicol. Res. 2016; 5:530-38.

15.- Peres LA, Cunha Júnior AD, Schäfer AJ, Silva AL, Gaspar AD, Scarpari DF, et al. Biomarkers of acute kidney injury. J. Bras. Nefrol. 2013; 35:229-36.

16.- Vaidya VS, Ferguson MA, Bonventre JV. Biomarkers of acute kidney injury. Annu. Rev. Pharmacol. Toxicol. 2008; 48:463-93.

17.- Coca SG, Yalavarthy R, Concato J, Parikh CR. Biomarkers for the diagnosis and risk stratification of acute kidney injury: a systematic review. Kidney Int. 2008; 73:1008-16.

18.- Takeda M, Khamdang S, Narikawa S, Kimura H, Hosoyamada M, Cha SH, et al. Characterization of methotrexate transport and its drug interactions with human organic anion transporters. J. Pharmacol. Exp. Ther. 2002; 302:666-71.

19.- Hagos Y, Wolff NA. Assessment of the role of renal organic anion transporters in drug-induced nephrotoxicity. Toxins 2010; 2:2055-82.

This article is protected by copyright. All rights reserved. 
20.- Schmid M, Dalela D, Tahbaz R, Langetepe J, Randazzo M, Dahlem R, et al. Novel biomarkers of acute kidney injury: evaluation and evidence in urologic surgery. World. J. Nephrol. 2015; 4:160-8.

21.- Abdeen A, Sonoda H, El-Shawarby R, Takahashi S, Ikeda M. Urinary excretion pattern of exosomal aquaporin-2 in rats that received gentamicin. Am. J. Physiol. Renal. Physiol. 2014; 307:F1227-37.

22.- du Cheyron D, Daubin C, Poggioli J, Ramakers M, Hovillier $\mathrm{P}$, Charbonneau $\mathrm{P}$ et al. Urinary measurement of $\mathrm{Na}^{+} / \mathrm{H}^{+}$exchanger isoform 3 (NHE3) protein as new marker of tubule injury in critically ill patients with ARF. Am. J. Kidney Dis. 2003; 42:497-506.

23.- Koroshi A. Microalbuminuria, is it so important? HippoKratia 2007; 11:105-7.

24.- Devarajan P, Murray P. Biomarkers in acute kidney injury: are we ready for prime time? Nephron Clin. Pract. 2014; 127:176-79.

25.- McMahon GM, Waikar SS. Biomarkers in nephrology: Core Curriculum 2013. Am. J. Kidney Dis. $2013 ; 62: 165-78$.

26.- Andreucci M, Faga T, Riccio E, Sabbatini M, Pisani A, Michael A. The potential use of biomarkers in predicting contrast-induced acute kidney injury. Int. J. Nephrol. Renovasc. Dis. 2016; 9:20521.

27.- Inoue K, Yuasa H. Molecular basis for pharmacokinetics and pharmacodynamics of methotrexate in rheumatoid arthritis therapy. Drug Metab. Pharmacokinet. 2014; 29:12-19.

28.- Majumdar S, Aggarwal BB. Methotrexate suppresses NF-kappaB activation through inhibition of IkappaBalpha phosphorylation and degradation. J. Immunol. 2001; 167:2911-20.

29.- Li SH, Hawthorne VS, Neal CL, Sanghera S, Xu J, Yang J et al. Upregulation of neutrophil gelatinase-associated lipocalin by ErbB2 through nuclear factor-kappaB activation. Cancer Res. 2009; 69:9163-68.

This article is protected by copyright. All rights reserved. 
30.- Arab HH, Salama SA, Maghrabi IA (2018) Camel milk attenuates methotrexate-induced kidney injury via activation of PI3K/Akt/eNOS signaling and intervention with oxidative aberrations. Food Funct. 2018; 23: 2661-2672.

31.- Li X, Abe E, Yamakawa Y, Yoneda G, Fujino R, Yamashita M et al. Effect of Administration Duration of Low Dose Methotrexate on Development of Acute Kidney Injury in Rats. J. Kidney 2016; 2, 6 pages.

32.- Dalaklioglu S, Sahin P, Ordueri EG, Celik-Ozenci C, Tasatargil A. Potential role of poly (ADP-ribose) polymerase (PARP) activation in methotrexate-induced nephrotoxicity and tubular apoptosis. Int. J. Toxicol. 2012; 31:430-440.

33.- Fouad AA, Al-Melhim WN. Vanillin mitigates the adverse impact of cisplatin and methotrexate on rat kidneys. Hum. Exp. Toxicol. 2018; 37:937-943.

34.-Carvalho Pedrosa D, Macedo de Oliveira Neves F, Cavalcante Meneses G, Pinheiro Gomes Wirtzbiki G, da Costa Moraes CA, Costa Martins AM, et al. Urinary KIM-1 in children undergoing nephrotoxic antineoplastic treatment: a prospective cohort study. Pediatr. Nephrol. 2015; 30:2207-2213.

35.-Bárdi E, Bobok I, Olah AV, Olah E, Kappelmayer J, Kiss C. Cystatin C is a suitable marker of glomerular function in children with cancer. Pediatr. Nephrol. 2004; 19, 1145-1147.

36.-Barreto JN, McClanahan AL, Rule AD, Thompson CA, Frazee E. Incorporating cystatin C to predict methotrexate elimination in patients with CNS lymphoma and suspicious renal function. Case Rep. Hematol. 2018; 26, 7169897, 5 pages.

37.-Coca SG, Parikh CR. Urinary biomarkers for acute kidney injury: perspectives on translation. Clin. J. Am. Soc. Nephrol. 2008; 3:481-490.

38.- Alvarez S, Suazo C, Boltansky A, Ursu M, Carvajal D, Innocenti G et al. Urinary exosomes as a source of kidney dysfunction biomarker in renal transplantation. Transplant. Proc. 2013; 45:3719-23.

This article is protected by copyright. All rights reserved. 
39.- Erdbrügger U, Le TH. Extracellular Vesicles in Renal Diseases: More than Novel Biomarkers? J. Am. Soc. Nephrol. 2016; 27:12-26.

40.- Yang JC, Lin MW, Rau CS, Jeng SF, Lu TH, Wu YC, et al. Altered exosomal protein expression in the serum of NF-KB knockout mice following skeletal muscle ischemia-reperfusion injury. J. Biomed. Sci. 2015; 22:40.

41.- Severin MJ, Torres AM. Time course effects of methotrexate on renal handling of water and electrolytes in rats. Role of Aquaporin-2 and Na-K-2Cl-cotransporter. Toxicol. Lett. 2019; 311:27-36.

42.- Vardi N, Parlakpinar H, Ates B, Cetin A, Otlu A. The protective effects of Prunus armeniaca L (apricot) against methotrexate-induced oxidative damage and apoptosis in rat kidney. J. Physiol. Biochem. 2013; 69:371-381.

43.- Kirbas A, Cure MC, Kalkan $\mathrm{Y}$, Cure E, Tumkaya L et al. Effect of infliximab on renal injury due to methotrexate in rat. Iran J. Kidney Dis. 2015; 9:221-229.

44.- Hafez HM, Ibrahim MA, Ibrahim SA., Amin EF, Goma Wet al. Potential protective effect of etanercept and aminoguanidine in methotrexate-induced hepatotoxicity and nephrotoxicity in rats. Eur. J. Pharmacol. 2015; 5:1-12.

45.- Turner PV, Brabb T, Pekow C, Vasbinde MA. Administration of Substances to Laboratory Animals: Routes of Administration and Factors to Consider. J. Am. Assoc. Lab. Anim. Sci. 2011; 50:600-613.

46.- Campagno RV, Severin MJ, Nosetto EC, Brandoni A, Torres AM. Renal expression and urinary excretion of $\mathrm{Na}^{+} /$dicarboxylate cotransporter 1 (NaDC1) in obstructive nephropathy: a candidate biomarker for this pathology. Pflügers Arch. 2018; 470:1777-86.

47.- Pinches MD, Betts CJ, Bickerton SJ, Beattie L, Burdett LD, Thomas HT, et al. Evaluation of novel urinary renal biomarkers with a cisplatin model of kidney injury: effects of collection period. Toxicol. Pathol. 2012; 40:534-40.

This article is protected by copyright. All rights reserved. 
48.- Romero-Calvo I, Ocón B, Martínez-Moya P, Suárez MD, Zarzuelo A, Martínez-Augustin O, de Medina FS. Reversible Ponceau staining as a loading control alternative to actin in Western blots. Anal. Biochem. 2010; 401:318-20.

49.- Bulacio RP, Nosetto EC, Brandoni A, Torres AM. Novel finding of caveolin-2 in apical membranes of proximal tubule and first detection of caveolin-2 in urine: A promising biomarker of renal disease. J. Cell. Biochem. 2019; 120:4966-74.

50.- Hazelhoff MH, Bulacio RP, Chevalier A, Torres AM. Renal expression of organic anion transporters is modified after mercuric chloride exposure: Gender-related differences. Toxicol. Lett. 2018; 295:390-96.

\section{FIGURE LEGENDS}

Figure 1. Urinary excretion of Oat5. Oat5 abundance in urine in Control and treated animals: M2D, M4D, M8D and M14D. Densitometric quantifications of Oat5 are expressed in bars (A) and as a time line (B).

Figure 2. Urea and creatinine plasma levels and clearance of creatinine in comparison with uOat5. Urea (g/L) (A) and creatinine plasma levels $(\mathrm{mg} / \mathrm{L})$ (B) and renal clearance of creatinine $(\mathrm{mL} / 24 h / 100 \mathrm{~g}$ b.w.) (C) (left $y$ axis, continuous line) and in uOat5 (right $y$ axis, dashed line) in Control and treated animals: M2D, M4D, M8D and M14D.

Figure 3. Renal Morphology. Optical microscopy photos of kidney histology in Control (A), M2D (B), M4D (C), M8D (D) and M14D (E) rats (Hematoxylin-eosin staining). Tubular dilatation (arrow), flattened tubular epithelium (star) and luminal debris (arrow head). Tubular alterations score (F). Bars $40 \mu \mathrm{m}$.

This article is protected by copyright. All rights reserved. 
Figure 4. Urinary AP activity, MAU and uNGAL in comparison with uOat5. Urinary AP activity (UI/g creatinine) (A), MAU (mg/g creatinine) (B), uNGAL (\%) (C) (left $y$ axis, continuous line) and in uOat5 (right $y$ axis, dashed line) in Control and treated animals: M2D, M4D, M8D and M14D.

Figure 5. Renal expression and immunolabeling of Oat5. Western blotting for Oat5 in renal homogenates and apical membranes (A). Kaleidoscope-prestained standards of molecular mass (Mr) corresponding to bovine serum albumin $(89.4 \mathrm{kDa})$ and to carbonic anhydrase (38.9 kDa). Immunohistochemistry for Oat5 (B) in Control and treated animals: M2D and M4D. Oat5 labeling was associated with the apical plasma membranes in proximal tubule cells (arrows). Bars $40 \mu \mathrm{m}$.

Figure 6. Oat5 in different urinary fractions. Oat5 abundance in Control and treated animals: M2D, M4D, M8D and M14D (A) or Control and M360 2D group (B) in total urine, exosomes (EXO) and supernatants (SN).

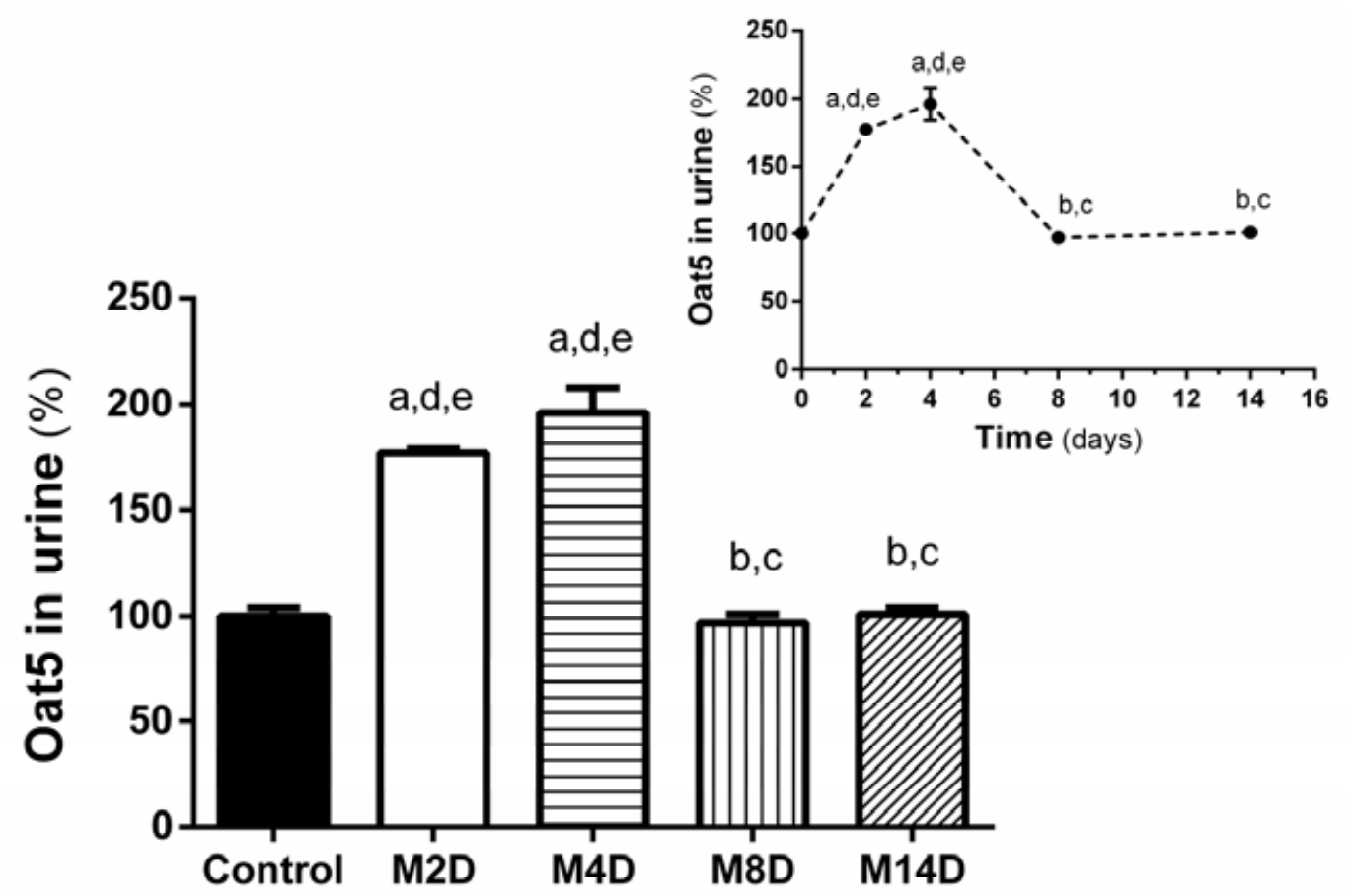

This article is protected by copyright. All rights reserved. 
A

$\Delta \quad$ Urea p

- Oat5 in urine

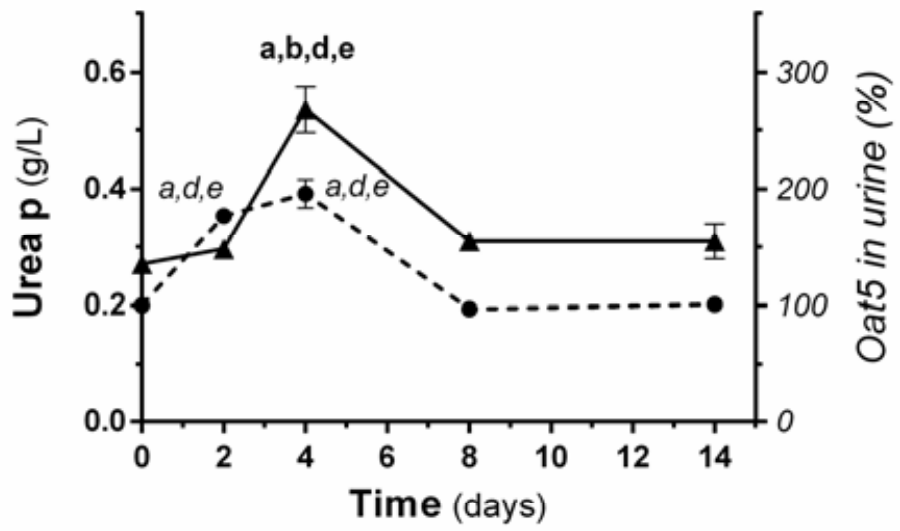

B

- Creatinine $\mathbf{p}$

$\bullet$ Oat5 in urine

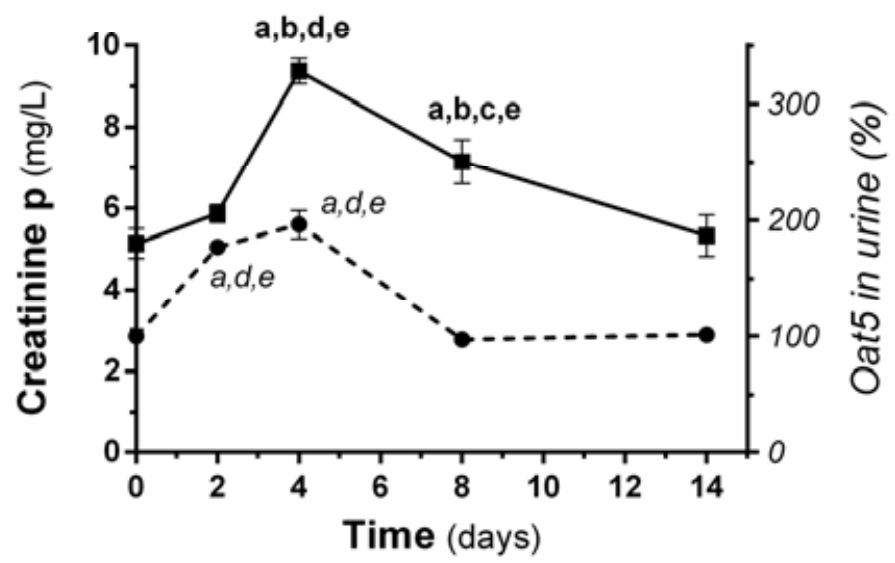

C

- Creatinine Clearance

- Oat5 in urine

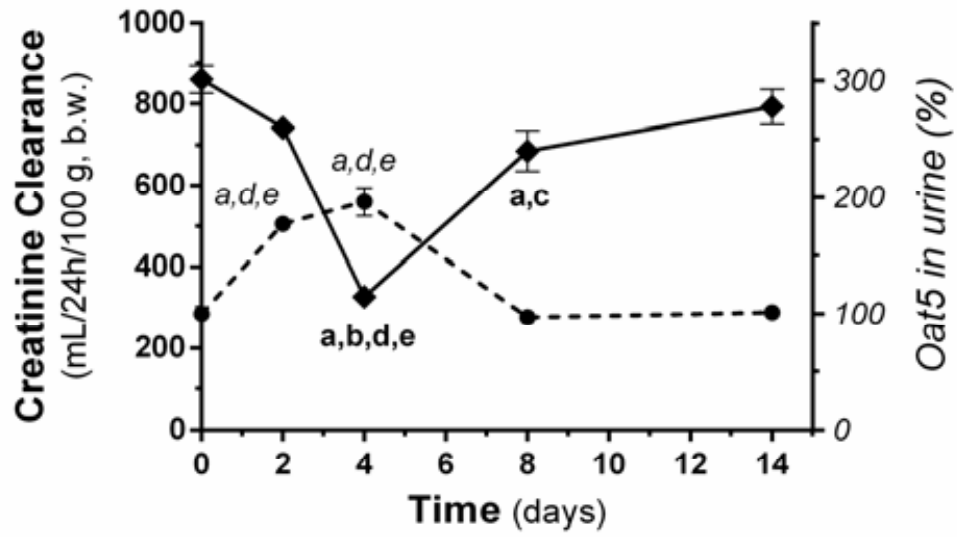

This article is protected by copyright. All rights reserved. 


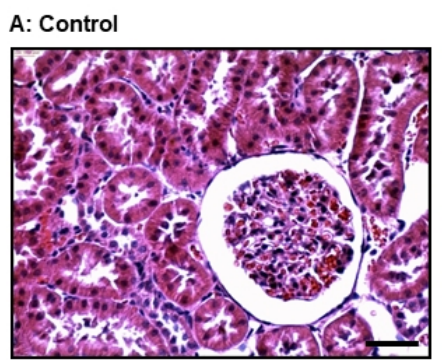

B: M2D

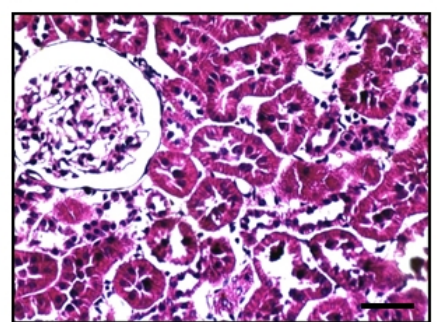

D: M8D

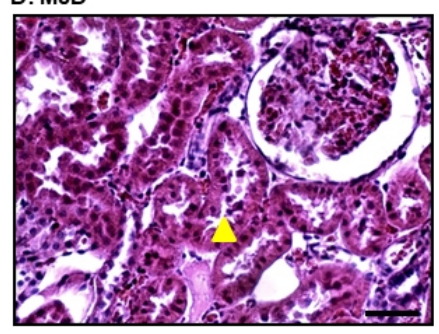

E: M14D

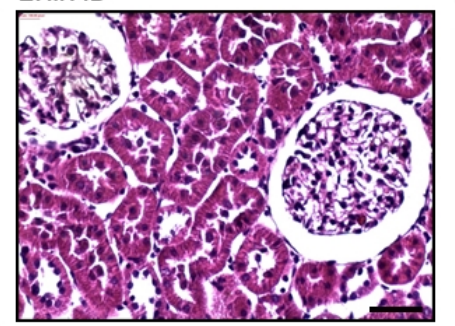

C: M4D

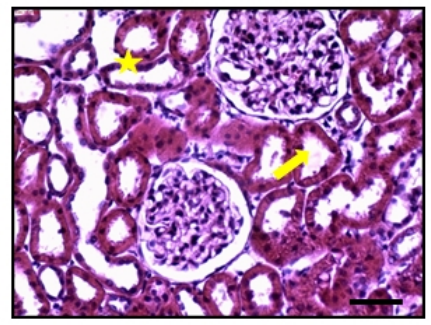

$\mathrm{F}$ :

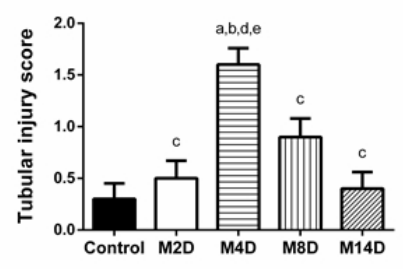

This article is protected by copyright. All rights reserved. 

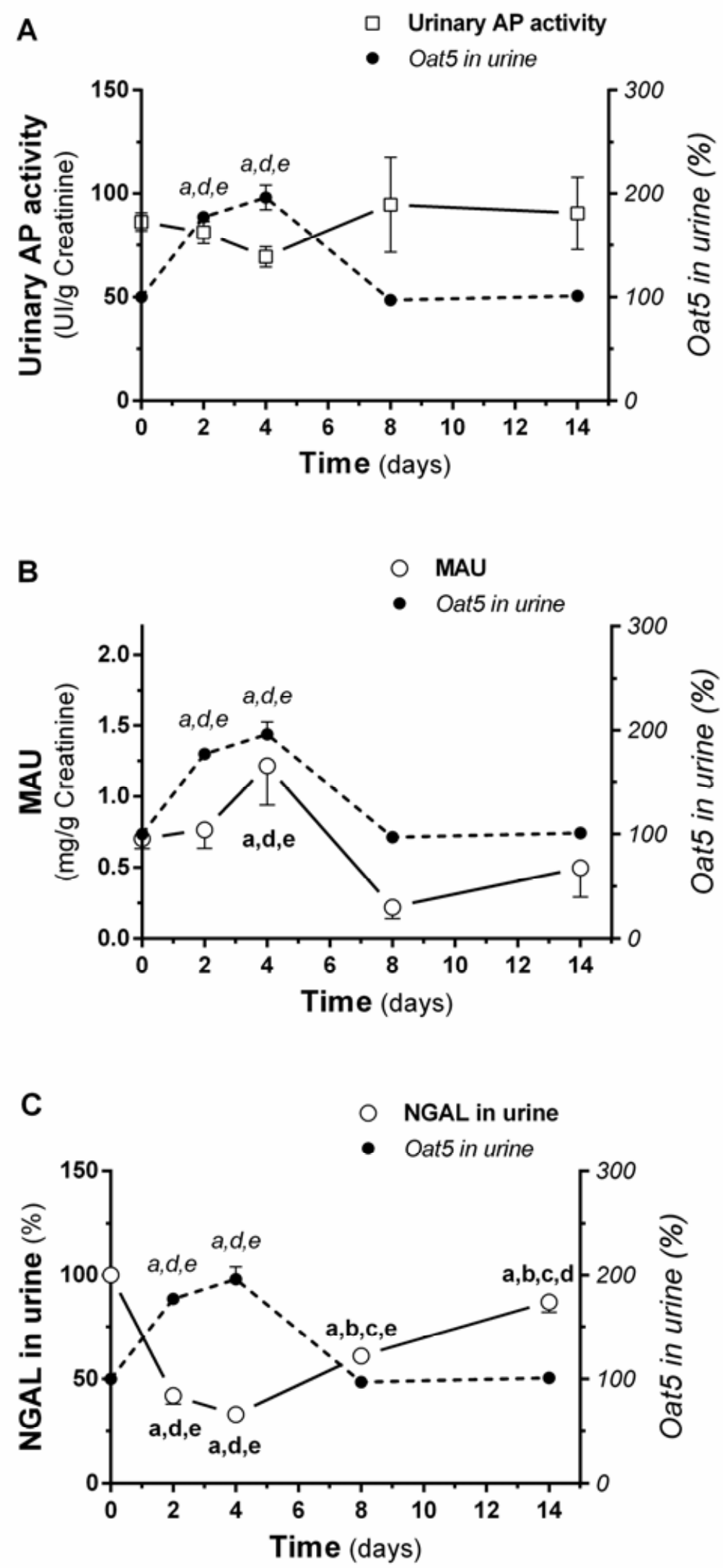

This article is protected by copyright. All rights reserved. 
A

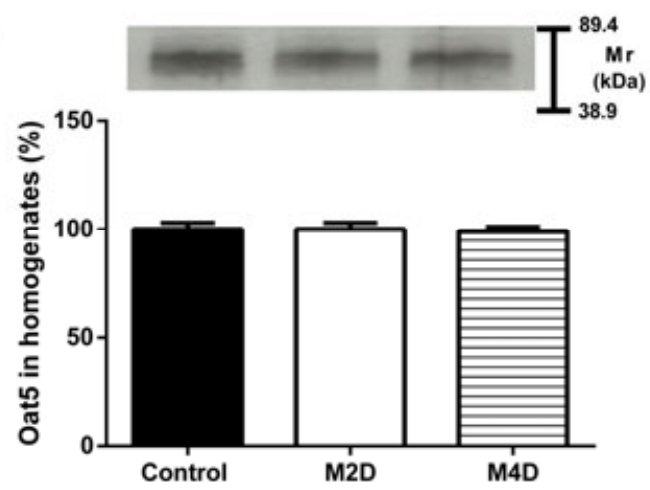

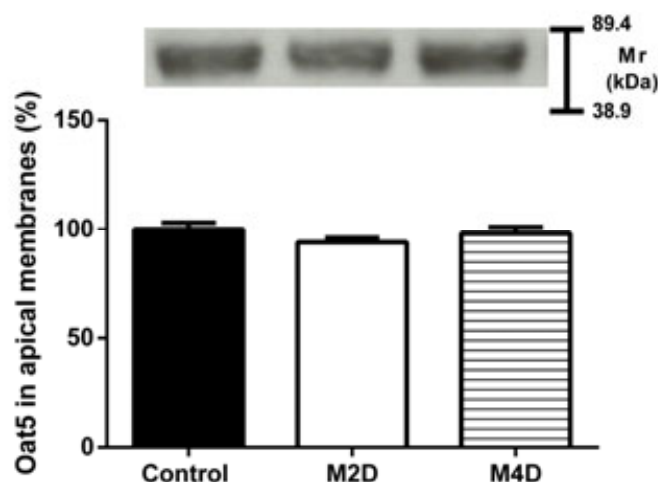

B

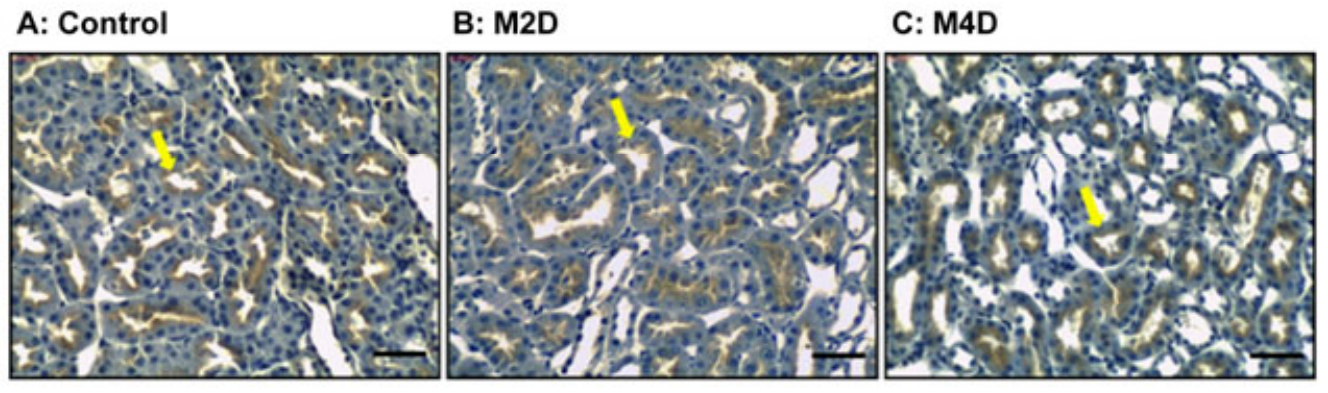


A

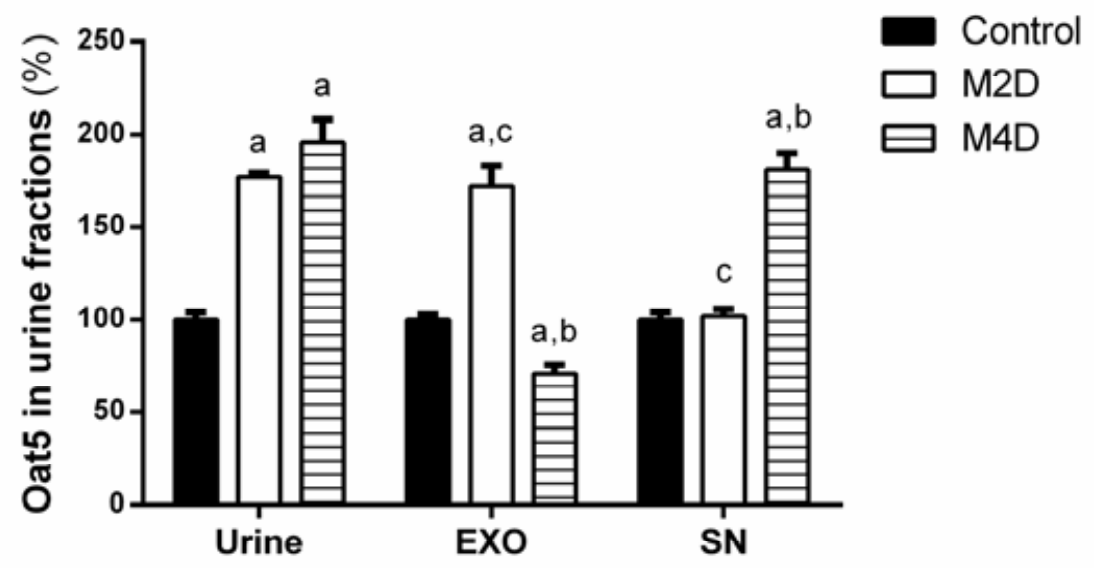

B

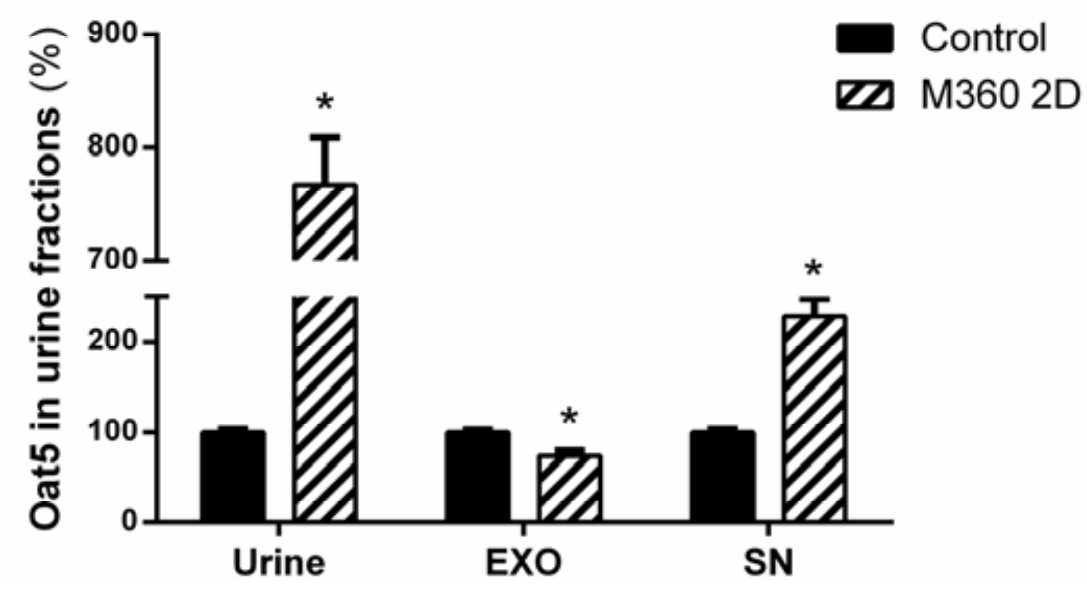

